\title{
A Novel Iterative Method Based on Bernstein-Adomian Polynomials to Solve Non-Linear Differential Equations
}

\author{
Afaf Nasser Yousif, Ahmed Farooq Qasim \\ College of Computer Sciences and Mathematics, University of Mosul, Mosul, Republic of Iraq \\ Email: afaf.nasser95@yahoo.com, ahmednumerical@uomosul.edu.iq
}

How to cite this paper: Yousif, A.N. and Qasim, A.F. (2020) A Novel Iterative Method Based on Bernstein-Adomian Polynomials to Solve Non-Linear Differential Equations. Open Access Library Journal, 7: e6267. https://doi.org/10.4236/oalib.1106267

Received: March 24, 2020

Accepted: April 26, 2020

Published: April 29, 2020

Copyright $\odot 2020$ by author(s) and Open Access Library Inc.

This work is licensed under the Creative Commons Attribution International License (CC BY 4.0).

http://creativecommons.org/licenses/by/4.0/

\section{(c) (i) Open Access}

\begin{abstract}
In this paper, a new iterative formula for solving ordinary and partial nonlinear differential equations is derived based on the combination between Bernstein's polynomial and the Adomian decomposition formula. The solution of the differential equations has been transformed into iterative formulas that find the solution directly without the need to convert it into a non-linear system of equations and solving it by other numerical methods that require considerable time and effort. The obtained results are compared with the exact solutions to show the efficiency and reliability of the proposed method which can be extended to solve a large variety of nonlinear differential equations. Tables are also given to show the variation of the absolute errors for larger approximation, namely for larger $n$.
\end{abstract}

\section{Subject Areas}

Applied Physics, Ordinary Differential Equation,

Partial Differential Equation

\section{Keywords}

Bernstein Polynomials, Adomian Decomposition Method, Differential Equations

\section{Introduction}

Differential equations are mostly prominent in the applications of chemistry, physics and mathematical models related to economic and biological processes, it is classified into two types, i.e. ordinary and partial, differential equations are divided into linear and non-linear, too. and there are many ways to solve this 
equation, such as Bernstein's polynomial which plays a prominent role in various branches of mathematics, and it has been used by many researchers to solve integral equations, and approximation theory [1] [2] [3] [4]. Saad N. AL-Azawi in [5] used Bernstein polynomials, for solving systems of the one-dimensional volterra linear integral equations of the second kind. Mohammed A. S. and Ishak $H$. in [6] solved the nonlinear stiff system of ordinary differential equations by applying Bernstein's polynomial. Yadollah O. and Sara D. in [4] used Bernstien's polynomials application to solve nonlinear fredholm integro-differential equations. As well as Ahmed F. Q. and Ekhlass S. AL-Rawi in [7] presented modified Bernstein Polynomials with Adomian decomposition method for solving ordinary and Partial differential equations. Adomian decomposition is a method used to solve linear and non-linear equations, for example, partial differential equations, algebraic, differential, and many systems, integral-differential equations, delay differential equations, ... etc., this method gives approximate solutions and accurate calculations of linear and nonlinear differential and integral equations, and the solution can be achieved to any degree of approximation, the benefit of this method is that it provides us with a direct way to resolve issues, in other words, without the need for disturbances, planning, enormous calculations and any other transfers [8]. The researchers Abdul M. Wazwaz and Salah M. EL-sayed in [9] conducted a comparative study between new modification and the modified decomposition method by introducing a new modification of the adomian decomposition method. Wazwaz, Randolph R. and Jun-Sheng D. in [10] used Adomian decomposition method for solving the systems of the Volterra integral forms of the Lane-Emden equations. M. Almazmumy, F. A. Hendi, H. O. Bakodah, and H. Alzumi in [11] presented some modifications to the Adomian decomposition Method in Ordinary Differential Equations to solve problems of Initial Value. Lina S. and weiguo W. in [12] used improved Adomian decomposition method is employed to solve analytic approximate solutions of the coupled fractional Burgers equations and the single nonlinear fractional reaction-diffusion equation with nonlinear terms of any order.

In this paper, we derive a new formula for solving non-linear ordinary and partial differential equations, using bernstein's polynomial with the adomian decomposition formula. In section 2, the basic ideas of Adomian's polynomial and modified Bernstein's polynomial are described. Section 3, a new formula for solving ordinary and partial nonlinear differential equations will be derived based on the combination of Bernstein's polynomial and Adomian decomposition formulas. The results and comparisons of the numerical solutions are presented in section 4 , and concluding remarks are given in section 5 .

\section{Adomian and Bernstein Polynomials}

The non-linear function $N(u)$ can be expressed by means of a power series whose radio of convergence is infinite, that is [13]

$$
N(u)=\sum_{n=0}^{\infty} N^{(n)}(0) \frac{u^{n}}{n !},|u|<\infty .
$$


Assuming the above hypotheses, the series whose terms are the Adomian polynomials $\left\{A_{n}\right\}_{n=0}^{\infty}$ results to be a generalization of the Taylor's series

$$
N(u)=\sum_{n=0}^{\infty} A_{n}\left(u_{0}, u_{1}, \cdots, u_{n}\right)=\sum_{n=0}^{\infty} N^{(n)}\left(u_{0}\right) \frac{\left(u-u_{0}\right)^{n}}{n !} .
$$

Is worthy to note that (2) is a rearranged expression of the series (1), and note that, due to hypothesis, this series is convergent. Consider now, the parametrization proposed by G. Adomian in [13] given by

$$
u_{\lambda}(x, t)=\sum_{n=0}^{\infty} u_{n}(x, t) f^{n}(\lambda),
$$

where $\lambda$ is a parameter in $R$ and $f$ is a complex-valued function such that $|f|<1$. With this choosing of $f$ and using the hypotheses above stated, the series (3) is absolutely convergent.

Substituting (3) in (2), we obtain

$$
N\left(u_{\lambda}\right)=\sum_{n=0}^{\infty} N^{(n)}\left(u_{0}\right) \frac{\left(\sum_{j=1}^{\infty} u_{j}(x, t) f^{j}(\lambda)\right)^{n}}{n !} .
$$

where

$$
\sum_{j=1}^{\infty} u_{j}(x, t) f^{j}(\lambda)
$$

is the absolute convergence. We can rearrange $N\left(u_{\lambda}\right)$ in order to obtain the series of the form $\sum_{n=0}^{\infty} A_{n} f^{n}(\lambda)$. Using (4) we can obtain the coefficients $A_{k}$ de $f^{k}(\lambda)$, and finally, we deduce the Adomian's polynomials. That is,

$$
\begin{gathered}
N\left(u_{\lambda}\right)=N\left(u_{0}\right)+N^{(1)}\left(u_{0}\right)\left(u_{1} f(\lambda)+u_{2} f^{2}(\lambda)+u_{3} f^{3}(\lambda)+\cdots\right) \\
+\frac{N^{(2)}\left(u_{0}\right)}{2 !}\left(u_{1} f(\lambda)+u_{2} f^{2}(\lambda)+u_{3} f^{3}(\lambda)+\cdots\right)^{2} \\
+\frac{N^{(3)}\left(u_{0}\right)}{3 !}\left(u_{1} f(\lambda)+u_{2} f^{2}(\lambda)+u_{3} f^{3}(\lambda)+\cdots\right)^{3}+\cdots \\
N\left(u_{\lambda}\right)=N\left(u_{0}\right)+N^{(1)}\left(u_{0}\right) u_{1} f(\lambda)+\left(N^{(1)}\left(u_{0}\right) u_{2}+N^{(2)}\left(u_{0}\right) \frac{u_{1}^{2}}{2 !}\right) f^{2}(\lambda) \\
+\left(N^{(1)}\left(u_{0}\right) u_{3}+N^{(2)}\left(u_{0}\right) u_{1} u_{2}+N^{(3)}\left(u_{0}\right) \frac{u_{1}^{3}}{3 !}\right) f^{3}(\lambda)+\cdots \\
=\sum_{n=0}^{\infty} A_{n}\left(u_{0}, u_{1}, \cdots, u_{n}\right) f^{n}(\lambda)
\end{gathered}
$$

Using Equation (7) making $f(\lambda)=\lambda$ and taking derivative at both sides of the equation, we can make the following identification

$$
\begin{gathered}
A_{0}\left(u_{0}\right)=N\left(u_{0}\right) \\
A_{1}\left(u_{0}, u_{1}\right)=N^{\prime}\left(u_{0}\right) u_{1} \\
A_{2}\left(u_{0}, u_{1}, u_{2}\right)=N^{\prime}\left(u_{0}\right) u_{2}+\frac{u_{1}^{2}}{2 !} N^{\prime \prime}\left(u_{0}\right) \\
A_{3}\left(u_{0}, u_{1}, u_{2}, u_{3}\right)=N^{\prime}\left(u_{0}\right) u_{3}+N^{\prime \prime}\left(u_{0}\right) u_{1} u_{2}+\frac{u_{1}^{3}}{3 !} N^{\prime \prime \prime}\left(u_{0}\right)
\end{gathered}
$$




$$
A_{4}\left(u_{0}, \cdots, u_{4}\right)=u_{4} N^{\prime}\left(u_{0}\right)+\left(\frac{1}{2 !} u_{2}^{2}+u_{1} u_{3}\right) N^{\prime \prime}\left(u_{0}\right)+\frac{u_{1}^{2} u_{2}}{2 !} N^{\prime \prime \prime}\left(u_{0}\right)+\frac{u_{1}^{4}}{4 !} N^{(i v)}\left(u_{0}\right)
$$

Hence we have obtained equation:

$$
A_{n}\left(u_{0}, u_{1}, \cdots, u_{n}\right)=\frac{1}{n !} \frac{\mathrm{d}^{n}}{\mathrm{~d} \lambda^{n}}\left[N\left(\sum_{k=0}^{n} \lambda^{k} u_{k}\right)\right]_{\lambda=0} .
$$

The Bernstein polynomials of degree $n$ in the interval $x \in[a, b]$ are defined by

$$
B_{k, n}(x)=\left(\begin{array}{l}
n \\
k
\end{array}\right)(x-a)^{k}(b-x)^{n-k} \text { for } k=0,1, \cdots, n
$$

where

$$
\left(\begin{array}{l}
n \\
k
\end{array}\right)=\frac{n !}{k !(n-k) !}
$$

There are $n+1 n$ th-degree Bernstein polynomials. For mathematical convenience, we usually set $B_{k, n}=0$, if $k<0$ or $k>n$.

The Bernstein approximation $B_{n}^{f}$ to a function $f:[a, b] \rightarrow R$ is the polynomial [14].

$$
B_{n}^{f}(x)=\sum_{k=0}^{n} f\left(a+\frac{k}{n}(b-a)\right)\left(\begin{array}{l}
n \\
k
\end{array}\right)(x-a)^{k}(b-x)^{n-k}
$$

If the $2 m^{\text {th }}$ order derivative $f^{(2 m)}$ is bounded in the interval $(a, b)$ then for each $x \in(a, b)$ we have modified Bernstein polynomial [14].

$$
B_{n}^{f}(x)=f(x)+\sum_{j=2}^{2 m-1} \frac{f^{(j)}(x)}{j ! n^{j}} T_{n, j}(x)+O\left(\frac{1}{n^{m}}\right)
$$

where

$$
T_{n, j}(x)=\sum_{k}(k-n x)^{j}\left(\begin{array}{l}
n \\
k
\end{array}\right)(x-a)^{k}(b-x)^{n-k}
$$

where $T_{n, j}(x)$ is the $t$ th central moment of random variable with a binomial distribution with parameters $n$ and $x$. Clearly, $T_{n, 0}=1, T_{n, 1}=0$. It is well known that the sequence $\left\{T_{n, j}(x)\right\}$ satisfies the following recurrence

$$
T_{n, j+1}(x)=(x-a)(b-x)\left(T_{n, j}^{\prime}(x)+n j T_{n, j-1}(x)\right) .
$$

\section{Modified Bernstein-Adomian Polynomials Method (MBAPM)}

In this section, a new formula for solving ordinary and partial nonlinear differential equations will be derived based on the combination of Bernstein's polynomial and Adomian decomposition formulas.

Consider the nonlinear differential equation

$$
L_{u}+R_{u}+N_{u}=g(x)
$$

where $L$ is the highest-order derivative which is assumed to be invertible, $R$ is a 
linear differential operator of less order than $L, N$ is the nonlinear operator and $g$ is the source term. If we apply the operator $L^{-1}$ which is the inverse of the $L$ to the Equation (15), we get

$$
u=L^{-1}(g)-L^{-1}\left(R_{u}\right)-L^{-1}\left(N_{y}\right)
$$

Let us suppose the solution of the Equation (15):

$$
u(x)=\sum_{z=0}^{\infty} u_{z}(x)
$$

Now we will treat the nonlinear term $N_{u}$ in Equation (17) using the modified Bernstein polynomial developed in the period $x \in[a, b]$ as:

$$
\begin{aligned}
N(x)= & \sum_{k=0}^{n} \frac{1}{(b-a)^{n}} \cdot \frac{n !}{k !(n-k) !} \cdot(x-a)^{k} \cdot(b-x)^{n-k} \cdot N\left(a+\frac{k}{n}(b-a)\right) \\
& -\sum_{j=2}^{2 m-1} \frac{N^{(j)}(x)}{j ! n^{j}} \cdot T_{n, j}(x)
\end{aligned}
$$

Applying Taylor's formula to the term $N\left(a+\frac{k}{n}(b-a)\right)$ around the point

$$
\begin{aligned}
& x=a+\frac{k}{n}(b-a) \text { we get: } \\
& \qquad \begin{aligned}
N(x)= & \sum_{k=0}^{n} \frac{1}{(b-a)^{n}} \cdot \frac{n !}{k !(n-k) !} \cdot(x-a)^{k} \cdot(b-x)^{n-k} \\
& \cdot \sum_{i=0}^{r} \frac{d^{i} N\left(a+\frac{k}{n}(b-a)\right)}{i !} \cdot\left(x-a+\frac{k}{n}(b-a)\right)^{i} \\
& -\sum_{j=2}^{2 m-1} \frac{N^{(j)}(x)}{j ! n^{j}} \cdot T_{n, j}(x)
\end{aligned}
\end{aligned}
$$

By converting Taylor's boundary into an iterative formula based on the Adomian polynomial, we get:

$$
N_{u}=\sum_{z=0}^{\infty} A_{z}
$$

where

$$
\begin{aligned}
A_{z}= & \sum_{k=0}^{n} \frac{1}{(b-a)^{n}} \cdot \frac{n !}{k !(n-k) !} \cdot(x-a)^{k} \cdot(b-x)^{n-k} \\
& \cdot \frac{1}{z !} \frac{\mathrm{d}^{z}}{\mathrm{~d} \lambda^{z}}\left[N\left(\sum_{i=0}^{z} \lambda^{i} u_{i}\left(a+\frac{k}{n}(b-a)\right)\right)\right]_{\lambda=0} \\
& -\sum_{j=2}^{2 m-1} \frac{\mathrm{d}^{j}\left[\frac{1}{z !} \frac{\mathrm{d}^{z}}{\mathrm{~d} \lambda^{z}}\left[N\left(\sum_{i=0}^{z} \lambda^{i} u_{i}(x)\right)\right]_{\lambda=0}\right]}{\mathrm{d} x^{j}} \cdot T_{n, j}(x)
\end{aligned}
$$

Using the Equations (20)-(21), we get:

$$
\sum_{z=0}^{\infty} u_{z}=f-L^{-1}\left(R\left(\sum_{z=0}^{\infty} u_{z}\right)\right)-L^{-1}\left(\sum_{z=0}^{\infty} A_{z}\right)
$$

where $f$ is calculated from the source term and the given condition(s) which are 
assumed to be prescribed. We now construct the recursive relation as:

$$
\begin{aligned}
& u_{0}=f \\
& u_{1}=-L^{-1} R\left(u_{0}\right)-L^{-1}(A) \\
& \quad \vdots \\
& u_{m+1}=-L^{-1}\left(R\left(u_{m}\right)\right)-L^{-1}\left(A_{m}\right), k \geq 0
\end{aligned}
$$

It can be easily said that the solution is

$$
u=\lim _{m \rightarrow \infty}\left(\sum_{z=0}^{m} u_{z}\right) .
$$

\section{Applications}

In this section, we apply our new formula to solve examples of nonlinear ordinary and partial differential equations; we adopt the following four examples.

\section{Example 1.}

Consider the first order nonlinear ordinary differential equation of the form [15]:

$$
\frac{\mathrm{d} u}{\mathrm{~d} x}-1=u^{2}(x),
$$

subject to the initial condition

$$
u_{(0)}=0
$$

Hence, the exact solution of Equation (22) is given by

$$
u(x)=\tan (x)
$$

Now, using Equation (21), we have $n=3$, in interval $[0,1]$, that is $a=0$ and $b$ $=1$.

$$
\begin{gathered}
u_{0}=x \\
u_{1}=0.3333333333 x^{3} \\
u_{2}=0.2666666666 x^{5}-0.2592592592 x^{4} \\
+0.1152263375 x^{3}+0.01234567901 x^{2} \\
u_{3}=0.4603174603 x^{7}-0.8251028807 x^{6}+0.4378600824 x^{5} \\
-0.04275262926 x^{4}+0.02788192856 x^{3}+0.003764670022 x^{2} \\
u=\sum_{i=0}^{n} u_{i}=x+0.4764415994 x^{3}+0.7045267490 x^{5}-0.3020118885 x^{4} \\
+0.01611034903 x^{2}+0.4603174603 x^{7}-0.8251028807 x^{6}
\end{gathered}
$$

The absolute and mean square errors are presented in Table 1 and Figure 1.

\section{Example 2.}

Consider the first order initial value problem with $\sin (u)$ nonlinearity [16]:

$$
\begin{gathered}
\frac{\mathrm{d} u}{\mathrm{~d} x}-\sin (u(x))=0, \quad 0 \leq x \leq 1 \\
u(0)=c_{0} .
\end{gathered}
$$

The exact solution of this problem can be expressed as 


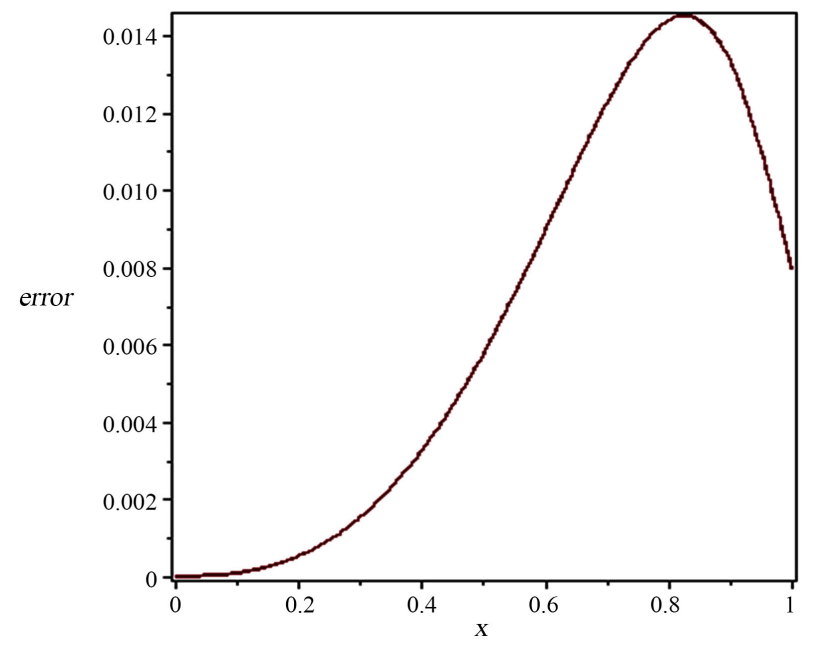

Figure 1. Illustrates the absolute error between the numerical solution and the exact solution when $n=5$.

Table 1. Comparison between MBAPM and the exact solution for example 1 when $m=2$.

\begin{tabular}{cll}
\hline $\boldsymbol{x}$ & Error $\boldsymbol{n}=\mathbf{3}$ & Error $\boldsymbol{n}=\mathbf{5}$ \\
\hline 0.1 & $2.789380 \mathrm{e}-4$ & $8.618620 \mathrm{e}-5$ \\
0.2 & $1.441226 \mathrm{e}-3$ & $5.219610 \mathrm{e}-4$ \\
0.3 & $3.742480 \mathrm{e}-3$ & $1.522006 \mathrm{e}-3$ \\
0.4 & $7.134112 \mathrm{e}-3$ & $3.245246 \mathrm{e}-3$ \\
0.5 & $1.112501 \mathrm{e}-2$ & $5.757043 \mathrm{e}-3$ \\
0.6 & $1.460750 \mathrm{e}-2$ & $8.928666 \mathrm{e}-3$ \\
0.7 & $1.575851 \mathrm{e}-2$ & $1.222704 \mathrm{e}-2$ \\
0.8 & $1.200522 \mathrm{e}-2$ & $1.438219 \mathrm{e}-2$ \\
0.9 & $2.419950 \mathrm{e}-4$ & $1.332488 \mathrm{e}-2$ \\
1 & $2.712634 \mathrm{e}-2$ & $7.928927 \mathrm{e}-3$ \\
Mse & $1.532554 \mathrm{e}-4$ & $7.227607 \mathrm{e}-5$ \\
\hline
\end{tabular}

$$
u(x)=2 \cot ^{-1}\left[\mathrm{e}^{x} \cot \left(\frac{c_{0}}{2}\right)\right]
$$

Now, using Equation (21), we have $n=3$, in interval $[0,1]$

$$
\begin{gathered}
u_{0}=1 \\
u_{1}=-0.8414709848 x \\
u_{2}=-2.50000000010^{-11} x^{4}+3.33333333310^{-11} x^{3}+0.2273243568 x^{2} \\
u_{3}=5.40302306110^{-12} x^{5}-1.87562980410^{-11} x^{4} \\
+0.05836258139 x^{3}-4.16959147010^{-12} x^{2} \\
u=\sum_{i=0}^{n} u_{i}=1-0.8414709848 x-4.3562980410^{-11} x^{4}+0.05836258142 x^{3} \\
+0.2273243568 x^{2}+5.40302306110^{-12} x^{5}
\end{gathered}
$$


We note from Table 1, Table 2 and Figure 1, Figure 2 that the mean square errors are $10^{-4}$ and increases when the value $\mathrm{n}$ increases until it reaches the exact solution when $n \rightarrow \infty$.

Example 3.

Consider the following hyperbolic nonlinear problem [17]:

$$
\frac{\partial u}{\partial t}=u \frac{\partial u}{\partial x} \text { in } 0<x \leq 1, \quad 0 \leq t \leq 1
$$

with the initial condition

$$
u(x, 0)=\frac{x}{10}, \quad 0<x \leq 1
$$

Equation (29) has the exact solution [17], $u(x, t)=\frac{-x}{t-10}$.

Now, using Equation (21), we have $n=3,[0,1]$

$$
\begin{gathered}
u_{0}=\frac{x}{10}=0.1 x \\
u_{1}=0.01 x t \\
u_{2}=0.001 x t^{2} \\
u_{3}=0.0001 x t^{3} \\
u=\sum_{i=0}^{n} u_{i}=\frac{1}{10} x+0.01 x t+0.001 x t^{2}+0.0001 x t^{3}
\end{gathered}
$$

\section{Example 4.}

Let us consider the Problem

$$
\frac{\partial u}{\partial t}=x^{2}-\frac{1}{4}\left(\frac{\partial u}{\partial x}\right)^{2}, 0<x \leq 1,0 \leq t \leq 1
$$

with the initial condition

$$
u(x, 0)=0, \quad 0<x \leq 1
$$

Equation (19) has the exact solution [17]:

$$
u(x, t)=x^{2} \tanh (t)
$$

Now, using Equation (21), we have $n=3,[0,1]$

$$
\begin{gathered}
u_{0}=x^{2} t \\
u_{1}=-0.3333333333 x^{2} t^{3} \\
u_{2}=0.1333333333 x^{2} t^{5} \\
u_{3}=-0.05396825396 x^{2} t^{7} \\
u=\sum_{i=0}^{n} u_{i}=x^{2} t-0.3333333333 x^{2} t^{3}+0.1333333333 x^{2} t^{5} \\
-0.05396825396 x^{2} t^{7}
\end{gathered}
$$

We note from Table 3, Table 4 and Figure 3, Figure 4 that the mean square errors are $10^{-11}$ and increases when the value $n$ increases until it reaches the exact solution when $n \rightarrow \infty$. 


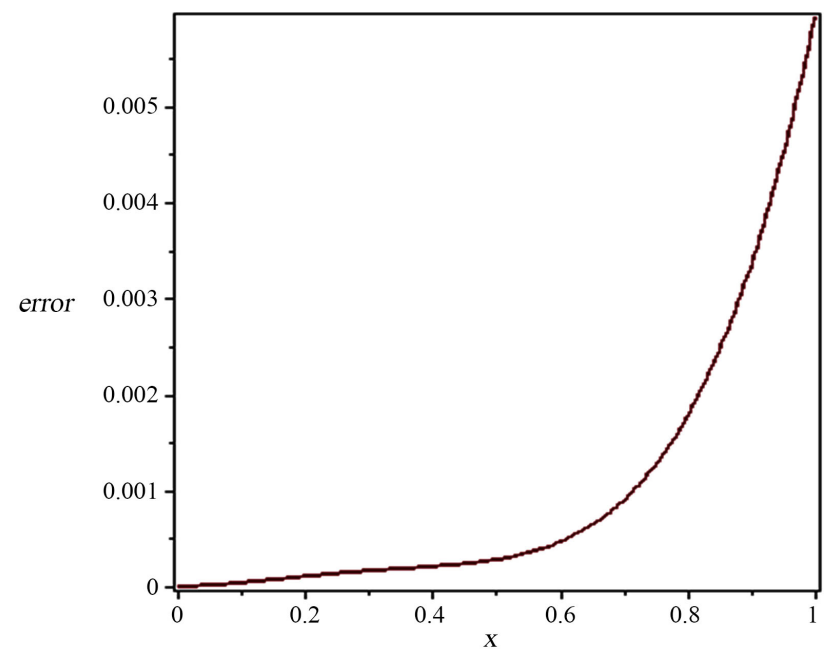

Figure 2. Illustrates the absolute error between the numerical solution and the exact solution when $n=5$.

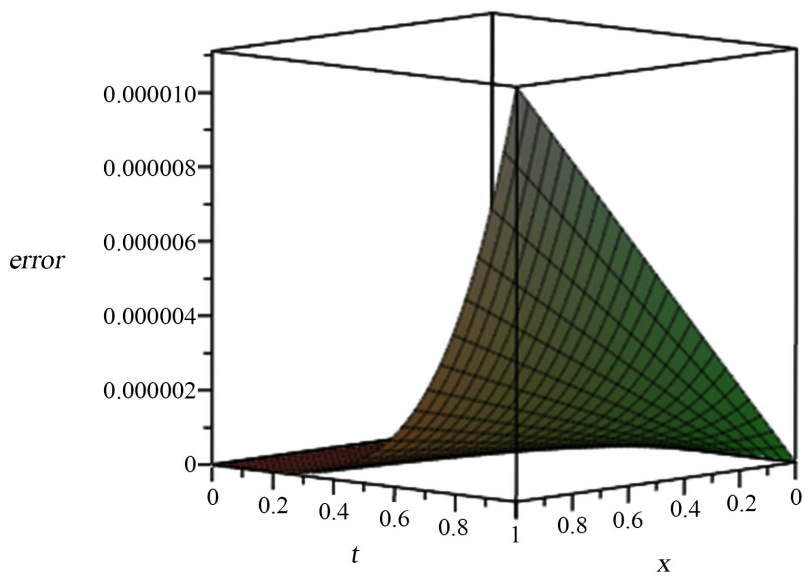

Figure 3. Illustrates the absolute error between the numerical solution and the exact solution when $n=3$.

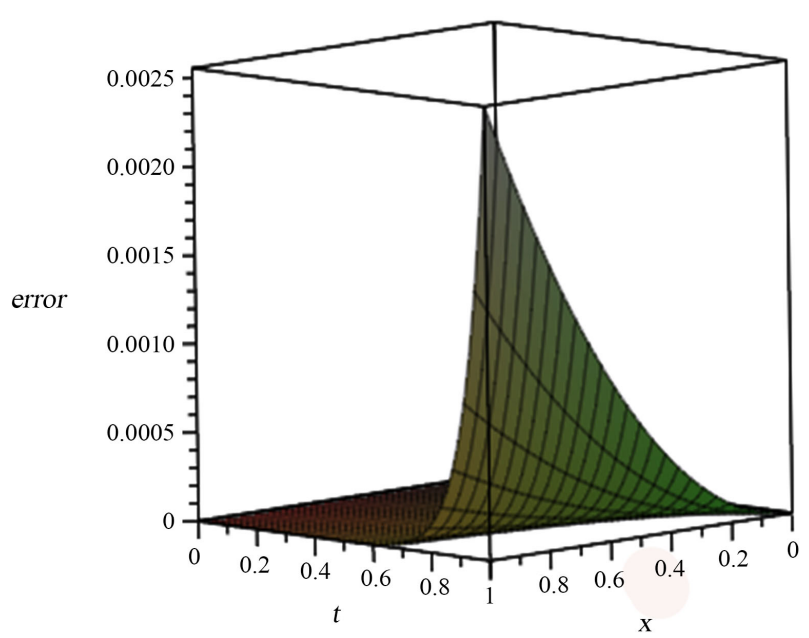

Figure 4. Illustrates the absolute error between the numerical solution and the exact solution when $n=5$. 
Table 2. Comparison between MBAPM and the exact solution for example 2 when $m=2$.

\begin{tabular}{cll}
\hline $\boldsymbol{x}$ & Error $\boldsymbol{n}=\mathbf{3}$ & Error $\boldsymbol{n}=\mathbf{5}$ \\
\hline 0.1 & $6.062700 \mathrm{e}-6$ & $3.577420 \mathrm{e}-5$ \\
0.2 & $9.525260 \mathrm{e}-5$ & $1.017902 \mathrm{e}-4$ \\
0.3 & $4.720114 \mathrm{e}-4$ & $1.595236 \mathrm{e}-4$ \\
0.4 & $1.456355 \mathrm{e}-3$ & $2.054227 \mathrm{e}-4$ \\
0.5 & $3.463354 \mathrm{e}-3$ & $2.773200 \mathrm{e}-4$ \\
0.6 & $6.982400 \mathrm{e}-3$ & $4.610532 \mathrm{e}-4$ \\
0.7 & $1.255787 \mathrm{e}-2$ & $8.956985 \mathrm{e}-4$ \\
0.8 & $2.077210 \mathrm{e}-2$ & $1.776480 \mathrm{e}-3$ \\
0.9 & $3.223112 \mathrm{e}-2$ & $3.354937 \mathrm{e}-3$ \\
1 & $4.755315 \mathrm{e}-2$ & $5.936313 \mathrm{e}-3$ \\
Mse & $3.952429 \mathrm{e}-4$ & $5.082233 \mathrm{e}-6$ \\
\hline
\end{tabular}

Table 3. Comparison between MBAPM and the exact solution for example 3 when $m=2$.

\begin{tabular}{|c|c|c|c|}
\hline$t$ & $x$ & Error $n=3$ & Error $n=5$ \\
\hline 0.1 & 0.1 & $1.000000 \mathrm{e}-10$ & 0 \\
\hline 0.2 & 0.2 & $2.000000 \mathrm{e}-10$ & 0 \\
\hline 0.3 & 0.3 & $3.000000 \mathrm{e}-10$ & 0 \\
\hline 0.4 & 0.4 & $4.000000 \mathrm{e}-10$ & 0 \\
\hline 0.5 & 0.5 & $5.000000 \mathrm{e}-10$ & 0 \\
\hline 0.6 & 0.6 & $6.000000 \mathrm{e}-10$ & $1.000000 \mathrm{e}-11$ \\
\hline 0.7 & 0.7 & $7.000000 \mathrm{e}-10$ & $1.000000 \mathrm{e}-11$ \\
\hline 0.8 & 0.8 & $8.000000 \mathrm{e}-10$ & $1.000000 \mathrm{e}-11$ \\
\hline 0.9 & 0.9 & $9.000000 \mathrm{e}-10$ & $1.000000 \mathrm{e}-11$ \\
\hline 1 & 1 & $1.000000 \mathrm{e}-9$ & 0 \\
\hline \multicolumn{2}{|c|}{ Mse } & $3.850000 \mathrm{e}-19$ & 0 \\
\hline
\end{tabular}

Table 4. Comparison between MBAPM and the exact solution for example 4 when $m=2$.

\begin{tabular}{|c|c|c|c|}
\hline$t$ & $x$ & Error $n=3$ & Error $n=5$ \\
\hline 0.1 & 0.1 & $2.000000 \mathrm{e}-13$ & 0 \\
\hline 0.2 & 0.2 & $1.000000 \mathrm{e}-12$ & 0 \\
\hline 0.3 & 0.3 & $2.000000 \mathrm{e}-12$ & 0 \\
\hline 0.4 & 0.4 & 0 & 0 \\
\hline 0.5 & 0.5 & $1.000000 \mathrm{e}-11$ & 0 \\
\hline 0.6 & 0.6 & 0 & $1.000000 \mathrm{e}-11$ \\
\hline 0.7 & 0.7 & 0 & $1.000000 \mathrm{e}-11$ \\
\hline 0.8 & 0.8 & $1.000000 \mathrm{e}-11$ & 0 \\
\hline 0.9 & 0.9 & $1.000000 \mathrm{e}-11$ & $1.000000 \mathrm{e}-11$ \\
\hline 1 & 1 & $2.000000 \mathrm{e}-11$ & 0 \\
\hline \multicolumn{2}{|c|}{ Mse } & $6.333250 \mathrm{e}-22$ & $1.740254 \mathrm{e}-23$ \\
\hline
\end{tabular}




\section{Conclusion}

In this study, a new formula based on modified Bernstein's polynomial has been derived to solve the ordinary and partial nonlinear differential equations by adding the Adomian decomposition formula to Bernstein's terms. The main benefit of this method is to convert the solution using Bernstein's polynomial from the method of converting a nonlinear differential equation to a nonlinear system of equations whose solution requires the use of other numerical methods such as fixed point, Newton-Raphson or other methods to iterative method that finds the solution directly as shown in the examples $(1-4)$. We use Bernstein expansions with Adomian decomposition method of the source term to obtain more accurate results. Figures 1-4 enable us to see that the difference between the numerical solutions and exact solutions by graphically. Tables are also given to show the variation of the absolute errors for larger approximation, namely for larger $n$. Maple 15 is used for calculations and sketching graphs.

\section{Acknowledgements}

The research is supported by College of Computer Sciences and Mathematics, University of Mosul, Republic of Iraq.

\section{Conflicts of Interest}

The authors declare no conflicts of interest regarding the publication of this paper.

\section{References}

[1] Yousefi, S.A., Barikbin, Z. and Dehghan, M. (2012) Ritz-Galerkin Method with Bernstein Polynomial Basis for Finding the Product Solution Form of Heat Equation with Non-Classic Boundary Conditions. International Journal of Numerical Methods for Heat, 22, 39-48. https://doi.org/10.1108/09615531211188784

[2] Maleknejad, K., Hashemizadeh, E. and Basirat, B. (2012) Computational Method Based on Bernstein Operational Matrices for Nonlinear Volterra-Fredholm-Hammerstein Integral Equations. Communications in Nonlinear Science and Numerical Simulation, 17, 52-61. https://doi.org/10.1016/j.cnsns.2011.04.023

[3] Tachev, G. (2012) Pointwise Approximation by Bernstein Polynomials. Bulletin of the Australian Mathematical Society, 85, 353-358. https://doi.org/10.1017/S0004972711002838

[4] Ordokhani, Y. and Far, S.D. (2011) Application of the Bernstein Polynomials for Solving the Nonlinear Fredholm Integro-Differential Equations. Journal of Applied Mathematics and Bioinformatics, 1, 13-31.

[5] AL-Azawi, S.N. (2011) Using Bernstein Polynomials for Solving Systems of Volterra Integral Equations of the Second Kind. Journal of the College of Basic Education, 17, 95-112.

[6] AL-Shbool, M. and Hashim, I. (2015) Bernstein Polynomials for Solving Nonlinear Stiff System of Ordinary Differential Equations. AIP Conference Proceedings, 1678, Article ID: 060015. https://doi.org/10.1063/1.4931342

[7] Qasim, A.F. and AL-Rawi, E.S. (2018) Adomian Decomposition Method with Mod- 
ified Bernstein Polynomials for Solving Ordinary and Partial Differential Equations. Journal of Applied Mathematics, 2018, Article ID: 1803107. https://doi.org/10.1155/2018/1803107

[8] Inc, M. (2004) On Numerical Solutions of Partial Differential Equations by the Decomposition Method. Kragujevac Journal of Mathematics, 26, 153-164.

[9] Wazwaz, A.M. and El-Sayed, S.M. (2001) A New Modification of the Adomian Decomposition Method for Linear and Nonlinear Operators. Applied Mathematics and Computation, 122, 393-405. https://doi.org/10.1016/S0096-3003(00)00060-6

[10] Wazwaz, A.M., Rach, R. and Duan, J.S. (2014) A Study on the Systems of the Volterra Integral Forms of the Lane-Emden Equations by the Adomian Decomposition Method. Mathematical Methods in the Applied Sciences, 37, 10-19.

https://doi.org/10.1002/mma.2776

[11] Almazmumy, M., Hendi, F.A., Bakodah, H.O. and Alzumi, H. (2012) Recent Modifications of Adomian Decomposition Method for Initial Value Problem in Ordinary Differential Equations. American Journal of Computational Mathematics, 20, 228. https://doi.org/10.4236/ajcm.2012.23030

[12] Song, L. and Wang, W. (2013) A New Improved Adomian Decomposition Method and Its Application to Fractional Differential Equations. Applied Mathematical Modelling, 37, 1590-1598. https://doi.org/10.1016/j.apm.2012.03.016

[13] González-Gaxiola, O. and Bernal-Jaquez, R. (2017) Applying Adomian Decomposition Method to Solve Burgess Equation with a Non-Linear Source. International Journal of Applied and Computational Mathematics, 3, 213-224. https://doi.org/10.1007/s40819-015-0100-4

[14] Gołębiewski, Z. and Cichoń, J. (2012) On Bernoulli Sums and Bernstein Polynomials. Discrete Mathematics and Theoretical Computer Science.

[15] Rawashdeh, M.S. and Maitama, S. (2015) Solving Nonlinear Ordinary Differential Equations Using the ND. Journal of Applied Analysis and Computation, 5, 77-88.

[16] Alkresheh, H.A. (2016) New Classes of Adomian Polynomials for the Adomian Decomposition Method. International Journal of Engineering Science Invention, 5 , 37-44.

[17] Bellman, R., Kashef, B.G. and Casti, J. (1972) Differential Quadrature: A Technique for the Rapid Solution of Nonlinear Partial Differential Equations. Journal of Computational Physics, 10, 40-52. https://doi.org/10.1016/0021-9991(72)90089-7 